\title{
Nambu-Jona-Lasinio model in a magnetic background: Size-dependent effects
}

\author{
L. M. Abreu, ${ }^{1}$ A. P. C. Malbouisson, ${ }^{2}$ and J. M. C. Malbouisson ${ }^{1}$ \\ ${ }^{1}$ Instituto de Física, Universidade Federal da Bahia, 40210-340, Salvador, BA, Brazil \\ ${ }^{2}$ Centro Brasileiro de Pesquisas Físicas, MCT, 22290-180, Rio de Janeiro, RJ, Brazil
}

(Received 31 May 2011; published 30 September 2011)

\begin{abstract}
We investigate finite-size effects on the chiral transition at finite temperature and density in the framework of the Nambu-Jona-Lasinio model in the presence of an external magnetic field. The thermodynamic potential and the gap equation are derived in the mean-field approximation and analyzed under the changing of the relevant parameters.
\end{abstract}

DOI: 10.1103/PhysRevD.84.065036

PACS numbers: 11.30.Qc, 12.38.Aw, 12.39.- $\mathrm{x}$

\section{INTRODUCTION}

In the framework of the standard model for fundamental interactions, the theory which is intended to account for processes involving strongly interacting matter is quantum chromodynamics (QCD). In high-energy phenomena, as for instance in deep inelastic scattering processes, the property of asymptotic freedom implies in a small effective coupling constant; then perturbative methods can be consistently employed. For several important situations, the theory can be perturbatively handled in the high-energy regime and important results have been obtained for a long time [1-3]. Distinctly, in the case of phenomena that would occur far from the high-energy domain, as the expected confiningdeconfining phase transition, perturbative methods are of little usefulness. Precise analytical studies are very difficult to be done in the low-energy regime of QCD, by reason of its very complex field-theoretical structure and of the fact that in this regime the effective coupling constant is large. In a field-theoretical context, there are a large number of, in some cases successful, attempts in the literature to bypass limitations of perturbation theory imposed by the need of smallness of the coupling constant. In particular, there are methods to perform resummations of perturbative series (even if they are divergent) using Borel transforms, under certain analyticity assumptions (the validity of the WatsonNevanlinna-Sokal theorem; see for instance Ref. [4] and other references therein). This amounts in some cases to analytically continuing the weak-coupling series to a strong-coupling domain, as for instance in Refs. [5-7]. However trials of application of these techniques to QCD did not give results of practical applicability. Perhaps because of these difficulties, one of the main methods employed in the strong-coupling regime of QCD is lattice calculation. Important results obtained with these techniques are described in detail for instance in Refs. [8-12]. Another relevant approach, namely, holographic QCD, has also been the subject of intensive studies over the last few years [13-15].

Because of this situation, a large amount of effort has been directed to construct simplified effective theories, sharing with QCD some of its basic properties. These have been largely employed as laboratories to get, analytically, insights on the behavior of hadronic matter. One of the most frequently used of these models is the NambuJona-Lasinio (NJL) model [16]. It is very useful for the investigation of dynamical symmetry breaking when the system is under certain conditions, like finite temperature, finite chemical potential, external magnetic field, and others [17-19]. In particular, magnetic effects are an object of recent interest, as for instance in Refs. [20-25]. These authors investigate the response of a quark-gluon plasma under the influence of an external magnetic field. They claim that an electromagnetic current is generated along the direction of the applied magnetic field, corresponding to the chiral magnetic effect. Magnetic effects on the quark-gluon plasma have been also investigated, for a relatively long time, in an astrophysical framework. For instance in Ref. [26] and other references therein, studies about the effect of strong magnetic fields on the quarkhadron phase transition at the core of neutron stars have been performed. There is a vast bibliography on the subject of the influence of electromagnetic fields on the formation of the quark-gluon plasma, particularly, studies performed using effective models for QCD. Some relevant examples of these works are in Refs. [27-45].

From a phenomenological viewpoint in effective theories for QCD, other effects also deserve investigation. In particular, an interesting aspect of the analysis of the phase transitions of NJL models is to study the changes induced by the finite size of the system on the phase diagrams, under the influence of a magnetic background. These effects of space compactification on four-fermion models, have been and still are the subject of intense investigation [46-58]. The general question is to estimate the relevance of the fluctuations due to finite-size effects in the phase diagram of the system, submitted to an external magnetic field. In this article, we extend the techniques introduced in Ref. [55] to investigate the finite-size effects on the dynamical symmetry breaking of the four-dimensional NJL model at finite temperature, chemical potential, and in the presence of an external magnetic background. We use zetafunction and compactification methods [59] which allows us in a direct way to determine analytically the size 
dependence of the effective potential and the gap equation. The zeta-function regularization technique has been very recently employed to calculate the magnetic susceptibility of the quark condensate and the polarization from chiral models [60]. Here, we study the influence of finite-size, external magnetic field, temperature, and chemical potential effects on the phase transition in the four-dimensional NJL model.

The organization of this paper is as follows. In Sec. II, we briefly review the NJL model and the zeta-function method to derive the effective potential in the mean-field approximation. Section III is devoted to analyzing the sizedependent effective potential and gap equation. Summary and concluding remarks are given in Sec. IV.

\section{THE MODEL}

Let us consider the massless four-dimensional NJL model in the presence of an external magnetic field, described by the Lagrangian density

$$
\mathcal{L}=\bar{q}(i \not \partial-Q \not A) q+G\left[(\bar{q} q)^{2}+\left(\bar{q} i \gamma_{5} \vec{\tau} q\right)^{2}\right],
$$

where $q$ and $\bar{q}$ are quark spinors carrying $N_{f}=2$ flavors and $N_{c}=3$ colors. The quantity $\vec{\tau}$ is a vector whose components are the generators in the flavor space, represented by the Pauli matrices, $Q$ stands for the electric charge of the quark fields $\left(Q_{u}=2 e / 3, Q_{d}=-e / 3\right)$, and $A^{\mu}$ is the four-potential associated with an external uniform magnetic field. We choose $A^{\mu}=\left(0,-x_{2} H, 0,0\right)$, with $H$ being constant, which means that we assume that the external magnetic field, $\mathbf{H}$, is parallel to the $z$ axis. Notice that the Lagrangian density $\mathcal{L}$ is invariant under global chiral transformations, i.e. $q \rightarrow \exp \left(i \theta \gamma_{5} \tau^{3} / 2\right) q$.

In order to study the phase structure of this model, it is convenient to perform a bosonization. We introduce auxiliary fields $\sigma$ and $\pi$, defined by $2 G \bar{q} q \equiv \sigma$ and $-2 G \bar{q} i \gamma^{5} \tau^{3} \equiv \pi=\pi^{3}$ (we assume $\pi^{1}=\pi^{2}=0$ ). In terms of the auxiliary fields, the Lagrangian density (1) becomes

$$
\tilde{\mathcal{L}}=\bar{q}\left(i \not \partial-Q \not A-\sigma-i \gamma^{5} \tau^{3} \pi\right) q-\frac{1}{4 G}\left(\sigma^{2}+\pi^{2}\right) .
$$

Then, integration over the fermion fields $q$ and $\bar{q}$ generates the effective action

$\Gamma_{\mathrm{eff}}(\sigma, \pi)=-\int d^{4} x \frac{1}{4 G} \sigma^{2}-\frac{i}{2} \operatorname{Tr} \ln \left(i \not \partial-Q \not A-\sigma-i \gamma^{5} \tau^{3} \pi\right)$,

where $\operatorname{Tr}$ means the operation of taking the trace over the color, flavor, Dirac, and coordinate spaces.

We will study the pure chiral sector $(\pi=0)$ and consider the mean-field approximation, which amounts to taking a uniform $\sigma$. In this case, the effective potential is obtained from Eq. (3) in the form

$$
U(\sigma)=\frac{\sigma^{2}}{4 G}+\frac{i}{2 V} \operatorname{Tr} \ln (i \not \partial-Q \not A-\sigma),
$$

where $V$ is the four-dimensional volume. The fermion field, minimally coupled to the external background magnetic field, obeys the Dirac equation

$$
(i \not \partial-Q \not A-\sigma) q=0 .
$$

Applying again the Dirac operator, it follows that each component of $q$ satisfies the equation

$$
\left[(i \partial+Q A)^{2}-\frac{Q}{2} \sigma^{\mu \nu} F_{\mu \nu}-\sigma^{2}\right] q=0,
$$

where $\sigma^{\mu \nu}=i\left[\gamma^{\mu}, \gamma^{\nu}\right] / 2$ and $F_{\mu \nu}=\partial_{\mu} A_{\nu}-\partial_{\nu} A_{\mu}$.

Now, due to the presence of the external magnetic field, with the gauge $A^{\mu}=\left(0,-x_{2} H, 0,0\right)$, the natural basis to expand the field operators is the set of the normalized eigenfunctions of the Landau basis. This means that the solutions of Eq. (6) should be written in the form [61,62]

$$
q(x)=e^{i\left(p_{0} x_{0}-p_{1} x_{1}-p_{3} x_{3}\right)} u\left(x_{2}\right),
$$

where $u\left(x_{2}\right)$ satisfies a harmonic oscillator equation,

$$
\begin{aligned}
& {\left[p_{2}^{2}+Q^{2} H^{2}\left(x_{2}-\frac{p_{1}}{Q H}\right)^{2}\right] u\left(x_{2}\right)} \\
& \quad=\left[p_{0}^{2}-p_{3}^{2}-\sigma^{2} \mp Q H\right] u\left(x_{2}\right) .
\end{aligned}
$$

The solutions of Eq. (8) are

$$
u_{n}\left(x_{2}\right)=\frac{1}{\sqrt{2^{n} n !}}\left(\frac{Q H}{\pi}\right)^{1 / 4} H_{n}\left(\sqrt{Q H}\left[x_{2}-\frac{p_{1}}{Q H}\right]\right) ;
$$

in the above equation $H_{n}$ are the Hermite polynomials and the corresponding energy spectrum provides the dispersion relation

$$
p_{0}^{2}=p_{3}^{2}+\sigma^{2}+(2 n+1-s) Q H,
$$

with $n=0,1,2, \ldots$, corresponding to the Landau levels, and $s= \pm 1$.

Notice that the introduction of the Landau basis implies a change in momentum space integrations of the type

$$
\int \frac{d^{4} p}{(2 \pi)^{4}} f(p) \rightarrow \frac{|Q| H}{2 \pi} \sum_{s= \pm 1} \sum_{n=0}^{\infty} \int \frac{d^{2} p}{(2 \pi)^{2}} f\left(p_{0}, p_{3}, n, s\right) ;
$$

this change, together with the results from Eq. (5) up to Eq. (10), allows us to rewrite Eq. (4) in the form

$$
\begin{aligned}
U(\sigma)= & \frac{\sigma^{2}}{4 G}+\frac{i}{2} \operatorname{tr} \frac{|Q| H}{2 \pi} \sum_{s= \pm 1} \sum_{n=0}^{\infty} \int \frac{d^{2} p}{(2 \pi)^{2}} \\
& \times \ln \left[p_{0}^{2}-p_{3}^{2}-\sigma^{2}-(2 n+1-s) Q H\right],
\end{aligned}
$$

where tr means the trace over the color and flavor spaces.

We wish to take into account simultaneously magnetic, finite-temperature, finite-chemical potential, and finite-size effects on the phase structure of the model. Magnetic effects are accounted for by the introduction of the 
Landau basis. For finite-temperature, finite-chemical potential, and finite-size effects, we consider a Euclidean space, with imaginary time and the spatial coordinate that remains after introduction of the Landau basis being compactified. We denote the Euclidean coordinate vectors by $x_{E}=\left(x_{0}, x_{1}, x_{2}, x_{3}\right)$, with $x_{0} \in[0, \beta]$ and $x_{3} \in[0, L]$, where $\beta$ is the inverse temperature, $\beta=T^{-1}$, and $L$ is the size of the compactified spatial dimension (the thickness of the system). This corresponds to the generalized Matsubara prescription,

$\int \frac{d^{2} p_{E}}{(2 \pi)^{2}} f\left(p_{0}, p_{3}, n, s\right) \rightarrow \frac{1}{\beta L} \sum_{l, m=-\infty}^{\infty} f\left(\omega_{l}, \omega_{m}, n, s\right)$,

where it is understood that, in the right-hand side, we have performed the replacements

$$
\begin{gathered}
p_{0} \rightarrow \omega_{l}=\frac{2 \pi}{\beta}\left(l+\frac{1}{2}\right)-i \mu ; \quad l=0, \pm 1, \pm 2, \ldots, \\
p_{3} \rightarrow \omega_{m}=\frac{2 \pi}{L}(m+c) ; \quad m=0, \pm 1, \pm 2, \ldots ;
\end{gathered}
$$

in the above equations $\mu$ is the chemical potential, $c=0$ and $c=\frac{1}{2}$ for periodic and antiperiodic spatial boundary conditions, respectively. In this paper we will restrict ourselves to antiperiodic boundary conditions for the spatial coordinates. The case of periodic spatial boundary conditions would follow along parallel lines. Unless explicitly stated, in all cases studied, the spatial boundary conditions are antiperiodic.

Using Eq. (12) in Eq. (11) we get, after some manipulations, the effective potential carrying magnetic, finitetemperature, and finite-size effects,

$$
\begin{aligned}
U(\sigma)= & \frac{\sigma^{2}}{4 G}-\frac{N_{c}}{2} \sum_{f} \frac{\left|Q_{f}\right| H}{2 \pi \beta L} \sum_{s= \pm 1} \sum_{n=0}^{\infty} \sum_{l, m=-\infty}^{\infty} \ln \left[\omega_{l}^{2}+\omega_{m}^{2}\right. \\
& \left.+\sigma^{2}+(2 n+1-s)\left|Q_{f}\right| H\right],
\end{aligned}
$$

where $\sum_{f}$ is the sum over the flavor indices.

The effective potential in Eq. (13) can be rewritten in terms of the Epstein generalized zeta functions, $Y(\eta)$, defined by

$Y(\eta)=\sum_{n=0}^{\infty} \sum_{l, m=-\infty}^{\infty}\left[\omega_{l}^{2}+\omega_{m}^{2}+\sigma^{2}+(2 n+1-s)\left|Q_{f}\right| H\right]^{-\eta}$,

that is,

$$
U(\sigma)=\frac{\sigma^{2}}{4 G}+\sum_{f} \sum_{s= \pm 1} \frac{N_{c}\left|Q_{f}\right| H}{4 \pi \beta L} Y^{\prime}(0),
$$

where the notation $Y_{\sigma}^{\prime}(0)$ means the derivative of $Y_{\sigma}(\eta)$ with respect to $\eta$, taken at $\eta=0$.

In order to obtain the effective potential in a more tractable form, we perform the analytical continuation of the Epstein generalized zeta function $Y_{\sigma}(\eta)$, to the whole complex $\eta$ plane. It reads, after use of recurrence formulas and some manipulations [55,56,59],

$$
\begin{aligned}
Y(\eta)= & \frac{\beta L}{2 \pi} \frac{\Gamma(\eta-1)}{\Gamma(\eta)} F_{1}(\eta-1)+\frac{\beta L}{\pi} \frac{1}{\Gamma(\eta)} F_{2}(\eta-1) \\
& +\frac{\beta}{\sqrt{\pi}} \frac{1}{\Gamma(\eta)} F_{3}\left(\eta-\frac{1}{2}\right) .
\end{aligned}
$$

The functions $F_{1}(\nu), F_{2}(\nu)$, and $F_{3}(\nu)$ are, respectively,

$$
\begin{aligned}
F_{1}(\nu)= & \left(\left|Q_{f}\right| H\right)^{-\nu}\left[\zeta\left(\nu, \frac{\sigma^{2}}{\left|Q_{f}\right| H}\right)-\frac{1}{2}\left(\frac{\sigma^{2}}{\left|Q_{f}\right| H}\right)^{-\nu}\right], \\
F_{2}(\nu)= & 2^{-\nu} \sum_{n=0}^{\infty} \sum_{m=1}^{\infty}\left(\frac{m L}{\sqrt{(2 n+1-s)\left|Q_{f}\right| H+\sigma^{2}}}\right)^{\nu}(-1)^{m} \\
& \times K_{\nu}\left(m L \sqrt{(2 n+1-s)\left|Q_{f}\right| H+\sigma^{2}}\right),
\end{aligned}
$$

and

$$
\begin{aligned}
F_{3}(\nu)= & 2^{1-\nu} \sum_{n=0}^{\infty} \sum_{m=-\infty}^{\infty} \sum_{l=1}^{\infty}\left(\frac{l \beta}{\sqrt{\frac{4 \pi^{2}}{L^{2}}\left(m+\frac{1}{2}\right)^{2}+(2 n+1-s)\left|Q_{f}\right| H+\sigma^{2}}}\right)^{\nu}(-1)^{l} \\
& \times \cosh (\mu \beta l) K_{\nu}\left(l \beta \sqrt{\frac{4 \pi^{2}}{L^{2}}\left(m+\frac{1}{2}\right)^{2}+(2 n+1-s)\left|Q_{f}\right| H+\sigma^{2}}\right),
\end{aligned}
$$

where $\zeta(\eta, a)$ is the Riemann zeta function and $K_{\eta}(z)$ is the modified Bessel function of the third kind.

Notice from Eq. (15) that the derivative of $Y_{\sigma}(\eta)$ with respect to $\eta$, for $\eta \rightarrow 0$, must be performed. This operation can be shortened by analyzing the pole structure of Eq. (16) for $\eta \rightarrow \epsilon(\epsilon \ll 1)$. Indeed, we see that

$$
\begin{aligned}
\frac{d}{d \eta}\left[\frac{\Gamma(\eta-1)}{\Gamma(\eta)} F_{1}(\eta)\right]_{\eta \rightarrow \epsilon} \approx & F_{1}(\epsilon-1)-F_{1}^{\prime}(\epsilon-1)-\epsilon F_{1}(\epsilon-1), \quad \frac{d}{d \eta}\left[\frac{1}{\Gamma(\eta)} F_{2}(\eta-1)\right]_{\eta \rightarrow \epsilon} \approx F_{2}(\epsilon-1), \\
& \frac{d}{d \eta}\left[\frac{1}{\Gamma(\eta)} F_{3}\left(\eta-\frac{1}{2}\right)\right]_{\eta \rightarrow \epsilon} \approx F_{3}\left(\epsilon-\frac{1}{2}\right) .
\end{aligned}
$$


Then, for $\epsilon \rightarrow 0$ we obtain the effective potential as

$$
\begin{aligned}
U(\sigma)= & \frac{\sigma^{2}}{4 G}+U_{\mathrm{vac}}-\sum_{f} \frac{N_{c}\left(\left|Q_{f}\right| H\right)^{2}}{2 \pi^{2}} F_{4}\left(\frac{\sigma^{2}}{2\left|Q_{f}\right| H}\right) \\
& +\sum_{f} \sum_{s= \pm 1} \frac{N_{c}\left|Q_{f}\right| H}{4 \pi^{2}}\left[F_{2}(-1)+\frac{\pi^{(1 / 2)}}{L} F_{3}\left(-\frac{1}{2}\right)\right],
\end{aligned}
$$

where $U_{\text {vac }}$ is the vacuum contribution, and

$$
F_{4}(z)=\left.\frac{\partial \zeta(\eta, z)}{\partial \eta}\right|_{\eta=-1}-\frac{1}{2}\left(z^{2}-z\right) \ln z+\frac{z^{2}}{4} .
$$

The contribution from the vacuum, $U_{\text {vac }}$, in Eq. (20) can be written as [20,24]

$$
\begin{aligned}
\frac{1}{N_{c}} U_{\mathrm{vac}}= & \frac{N_{f}}{8 \pi^{2}}\left[\sigma^{4} \ln \left(\frac{\Lambda+\sqrt{\Lambda^{2}+\sigma^{2}}}{\sigma}\right)\right. \\
& \left.-\Lambda\left(2 \Lambda^{2}+\sigma^{2}\right) \sqrt{\Lambda^{2}+\sigma^{2}}\right]
\end{aligned}
$$

where $\Lambda$ is a cutoff parameter. As pointed out in Ref. [60], neglecting contributions which depend on the magnetic field but not on the quark condensate, $U_{\text {vac }}$ is the unique ultraviolet divergent contribution for the quantum effective action.

Finally, now we are able to obtain explicitly the gap equation,

$$
\left.\frac{\partial U(\sigma)}{\partial \sigma}\right|_{\sigma=M}=0,
$$

where $M=M(T, \mu, L, H)$ is the $(T, \mu, L, H)$-dependent order parameter of the chiral symmetry breaking transition; equivalently $M$ plays the role of a dynamical fermion mass, such that when it has a nonvanishing value, the system is in the chiral broken phase. Insertion of Eq. (20) in (23), generates one trivial solution, $M=0$, and other, nontrivial ones, coming from the equation

$$
\begin{aligned}
\frac{1}{G} & -C_{\mathrm{vac}}(M, \Lambda)-\sum_{f} \frac{N_{c}\left(\left|Q_{f}\right| H\right)}{\pi^{2}} I\left(\frac{M^{2}}{\left|Q_{f}\right| H}\right) \\
& -\sum_{f} \sum_{s= \pm 1} \frac{N_{c}\left|Q_{f}\right| H}{\pi^{2}}\left[F_{2}(0)+\frac{\pi^{(1 / 2)}}{L} F_{3}\left(\frac{1}{2}\right)\right]=0,
\end{aligned}
$$

where

$$
I(z)=\ln \Gamma(z)-\frac{1}{2} \ln 2 \pi+z-\frac{1}{2}(2 z-1) \ln z,
$$

and

$$
\begin{aligned}
C_{\mathrm{vac}}(M, \Lambda)= & \frac{N_{c} N_{f}}{\pi^{2}}\left[\Lambda \sqrt{\Lambda^{2}+M^{2}}\right. \\
& \left.-M^{2} \ln \left(\frac{\Lambda+\sqrt{\Lambda^{2}+M^{2}}}{M}\right)\right] .
\end{aligned}
$$

It is worth mentioning that the sum over $s$ in Eqs. (20) and (24) can be explicitly performed, resulting in

$$
\sum_{n=0}^{\infty} \sum_{s= \pm 1} f(2 n+1-s) \rightarrow \sum_{n=0}^{\infty}\left(2-\delta_{n 0}\right) f(2 n)
$$

\section{PHASE STRUCTURE}

We now study the behavior of the effective potential and the solutions of the gap equations given in Eqs. (20) and (24) under the change of values of the relevant parameters of the model, looking specially at finite-size effects on the phase structure. In the figures, we use the notation already introduced and some other; for clarity, we recall that $M$ is the effective quark mass, $x=1 / L, T=1 / \beta, \omega=e H$ is the cyclotron frequency, $\mu$ is the chemical potential, and $G$ is the coupling constant. It is interesting to introduce the critical coupling, $G_{c}$; in absence of magnetic field, and for vanishing temperature and chemical potential, in free space, it is given by $G_{c}=\pi^{2} / N_{c} N_{f}=\pi^{2} / 6$ (see, for example, Ref. [20]). The region $G>G_{c}$ is the region with nontrivial mass. In what follows all physical quantities are scaled by the ultraviolet cutoff parameter $\Lambda$, which has to be determined by fitting to experimental data. Then we have,

$$
\begin{gathered}
U / \Lambda^{4} \rightarrow U, \quad M / \Lambda \rightarrow M, \quad x / \Lambda \rightarrow x, \quad T / \Lambda \rightarrow T, \\
\omega / \Lambda^{2} \rightarrow \omega, \quad \mu / \Lambda \rightarrow \mu, \\
1 / G \Lambda^{2} \rightarrow 1 / G .
\end{gathered}
$$

In Figs. 1-4, the effective potential and the solutions of the gap equation are plotted for the broken phase, taking

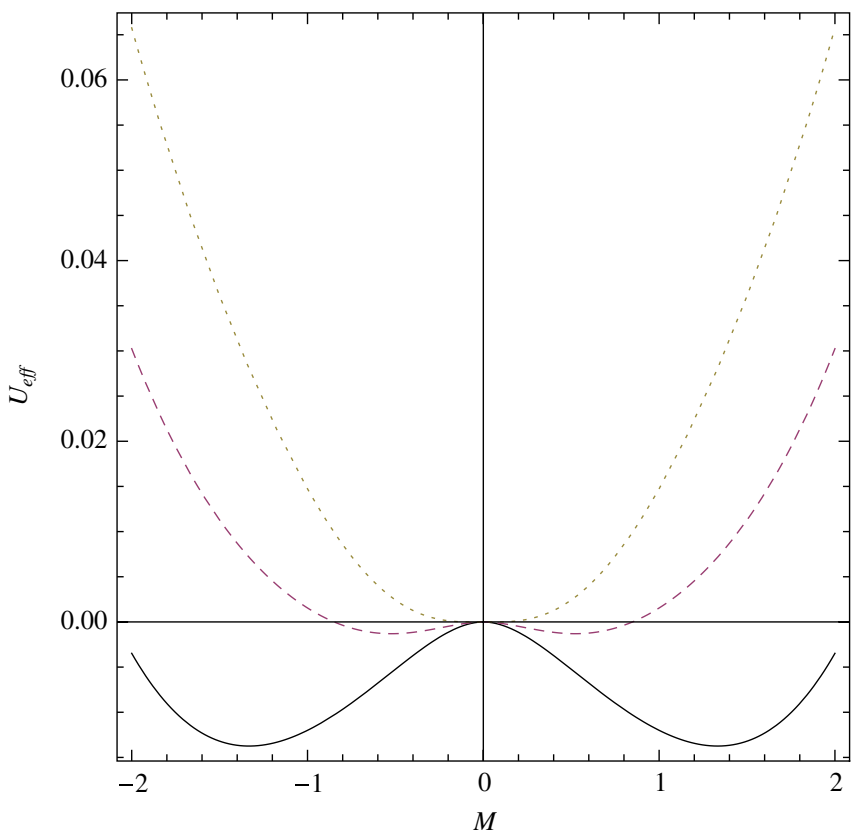

FIG. 1 (color online). Plot of effective potential, Eq. (20), for $x=0.01, \mu=0, G=\pi^{2} / 2$ and $\omega=0.1$. Solid, dashed, and dotted lines represent $T=0.6,0.7$, and 0.8 , respectively. 


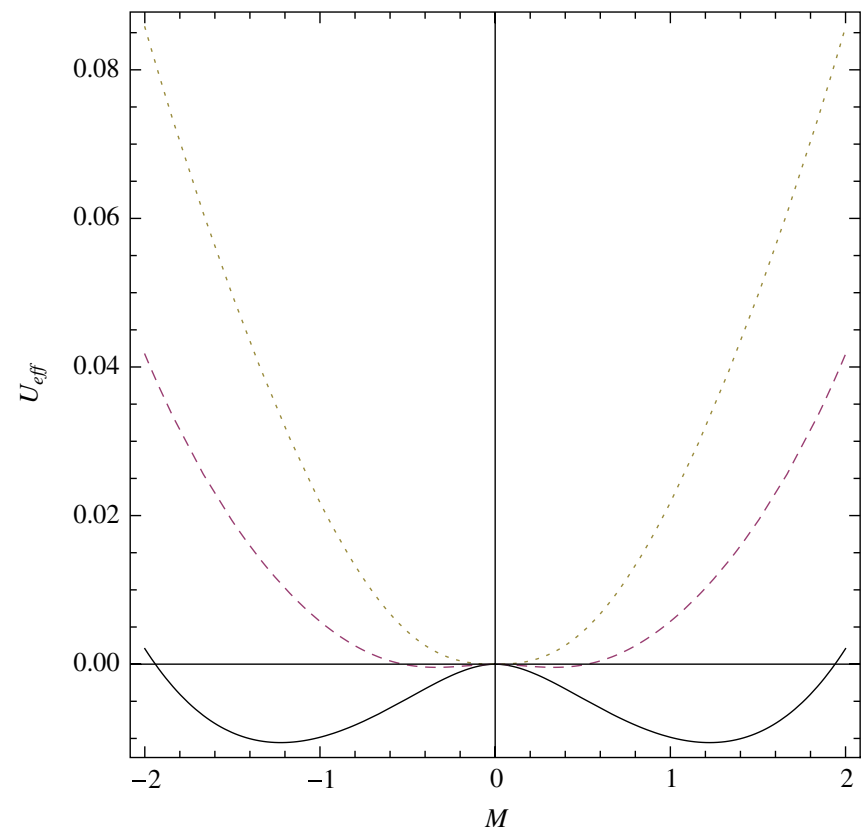

FIG. 2 (color online). Plot of effective potential, Eq. (20), for $T=0.01, \mu=0, G=\pi^{2} / 2$, and $\omega=0.1$. Solid, dashed, and dotted lines represent $x=0.6,0.7$, and 0.8 , respectively.

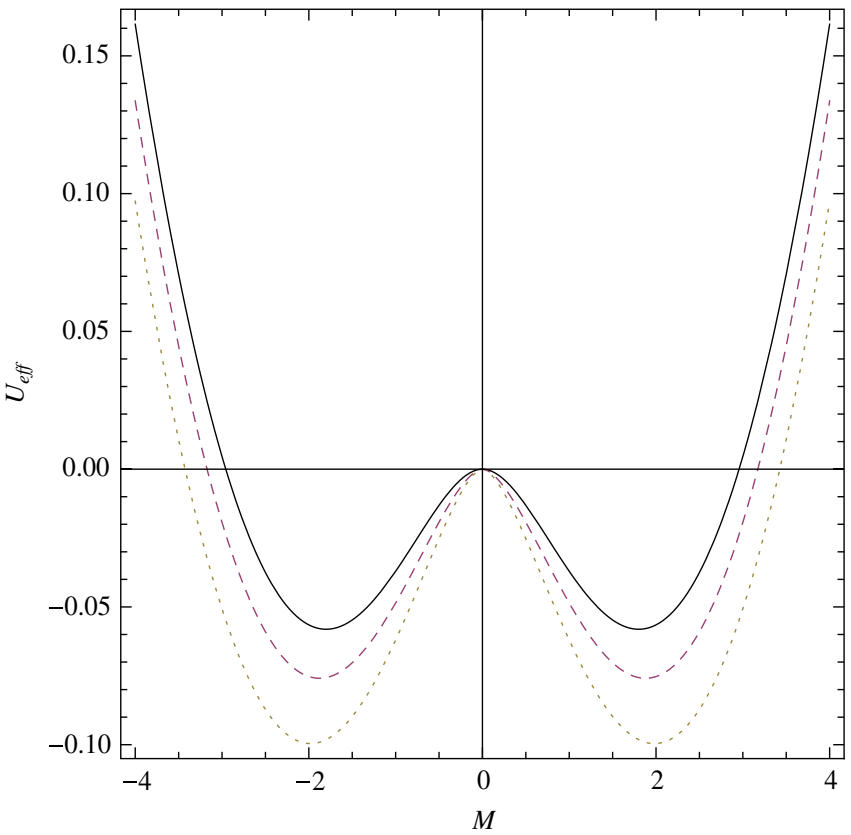

FIG. 3 (color online). Plot of effective potential, Eq. (20), for $T=0.4, \mu=0, G=\pi^{2} / 2$, and $x=0.01$. Solid, dashed, and dotted lines represent $\omega=0.1,1.0$, and 1.5 , respectively.

the coupling constant $G=\pi^{2} / 2>G_{c}$. Some relevant aspects should be noticed:

(i) For fixed values of $x, \mu$, and $\omega$, the broken phase is inhibited as the temperature increases, as expected.

(ii) On the other hand, for fixed values of $T, \mu$, and $\omega$, the chiral-condensate region diminishes as the size

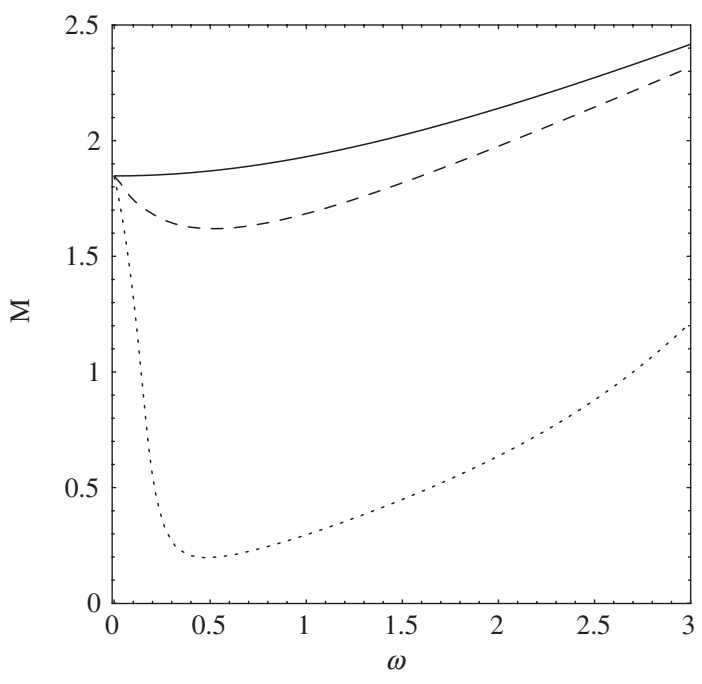

FIG. 4. Plot of effective quark mass $M$ versus magnetic field from Eq. (24) for $T=0.1, \mu=0.5$, and $G=\pi^{2} / 2$. Solid, dashed, and dotted lines represent $x=0.1,0.5$, and 0.7 , respectively.

of the system decreases. This means that, as the size of the system decreases, the maintenance of longrange correlations is inhibited, favoring disorder. This is a general feature of second-order phase transitions, occurring also, for example, in superconducting films, wires, and grains [63].

(iii) Another point is that for fixed values of $T, \mu$, and $x$, the broken phase is favored as the magnetic field is increased. This effect, known as magnetic catalysis, is also present in the system without boundaries [20,21,23-25]. The physical meaning of this effect is that the magnetic field drives the system to the ordered phase.

(iv) In Fig. 4 it is shown that the dependence of $M$ on the magnetic field exhibits a similar behavior to that of Refs. [20,24] for the smallest value of $x$, large $L: M$ increases with $\omega$. However, the dependence of $M$ on $\omega$ is modified as the size of the system diminishes: first, the mass decreases, reaches a minimum value, and then starts to grow as the field increases.

In Figs. 5-7 the effective potential and the solutions of the gap equation are plotted with coupling constant $G=\pi^{2} / 6$, i.e. its critical value in the absence of boundaries, at zero temperature, chemical potential, and magnetic field. The main conclusions coming out of the figures are:

(i) At fixed values of $T, \mu$, and $\omega$, there is a transition from the broken to the unbroken phase as the size of the system decreases.

(ii) For fixed values of $T, \mu$, and $x$, there is a transition from the unbroken to the broken phase as the magnetic field increases. As in the previous case, the increasing of the magnetic field tends to drive the system to the ordered phase. 


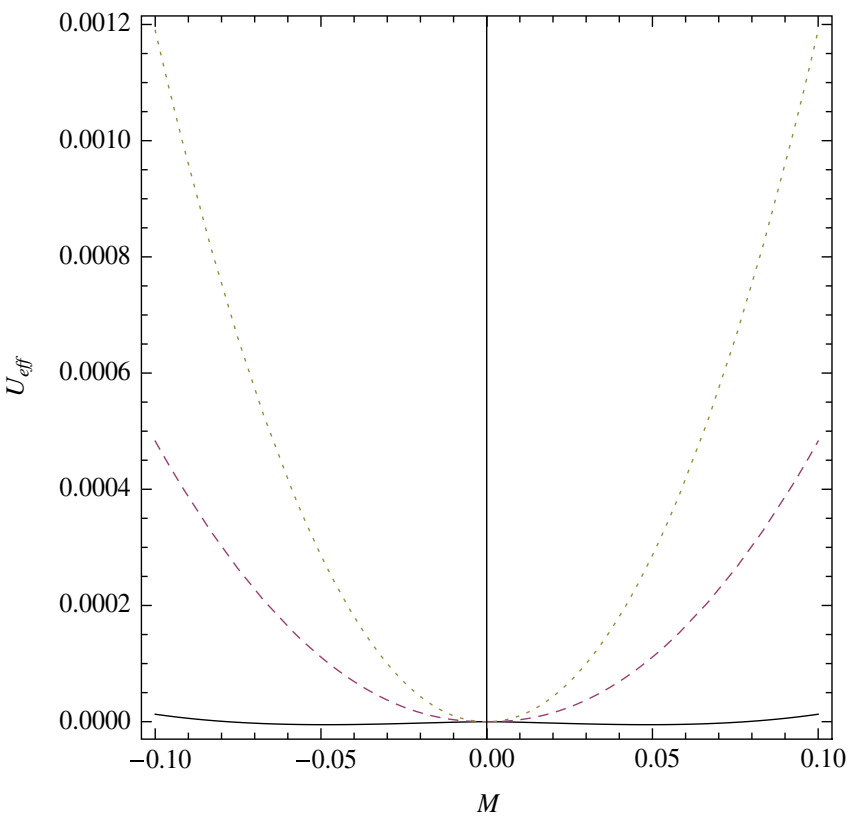

FIG. 5 (color online). Plot of effective potential, Eq. (20), for $T=0.1, \mu=0.1, G=\pi^{2} / 6$, and $\omega=0.1$. Solid, dashed, and dotted lines represent $x=0.1,0.5$ and 1.0, respectively.

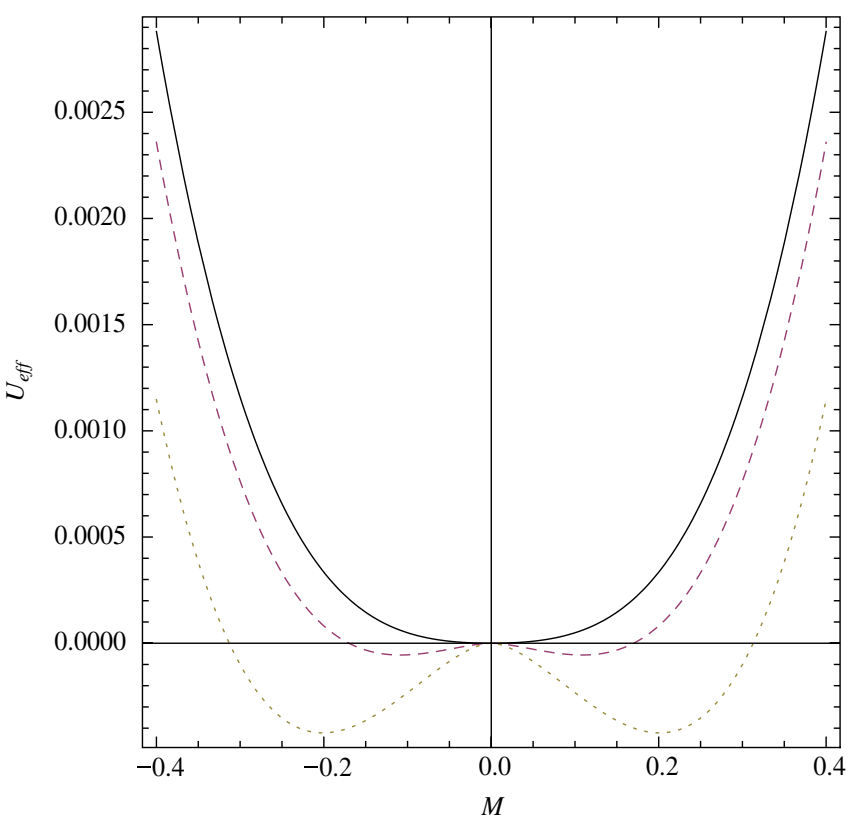

FIG. 6 (color online). Plot of effective potential, Eq. (20), for $T=0.1, \mu=0.0, G=\pi^{2} / 6$, and $x=0.1$. Solid, dashed, and dotted lines represent $\omega=0.01,0.2$, and 0.4 , respectively.

(iii) We see from Fig. 7 that $M$ assumes a vanishing value at $\omega, \mu, T \approx 0$, an expected result for the coupling constant at the critical value. Nevertheless, $M$ increases with magnetic field in all displayed cases of size of the system. However, for a given value of $\omega$, the corresponding values of $M$ are larger for larger values of $L$ (smaller values of $x$ ).

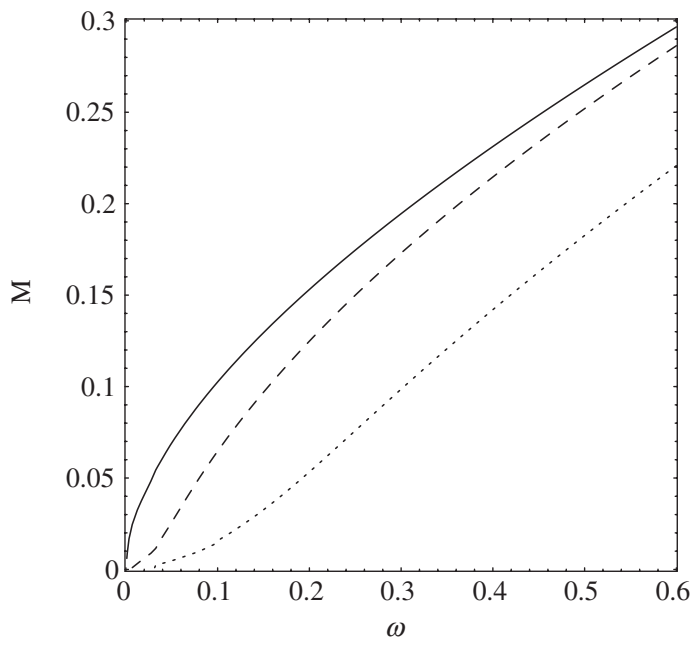

FIG. 7. Plot of effective quark mass $M$ versus magnetic field from Eq. (24) for $T=0.001, \mu=0$, and $G=\pi^{2} / 6$. Solid, dashed, and dotted lines represent $x=0.01,0.1$, and 0.2 , respectively.

In Figs. 8 and 9 the effective potential and the gap equation are plotted with coupling constant $G=\pi^{2} / 12$, smaller than the critical value $G_{c}$. We see from the figures that at fixed values of $T, \mu$, and $\omega$, there is a transition from the broken to the unbroken phase as the size of the system decreases, as it was in the previous case. In addition, it is shown that stronger values of the magnetic field are necessary to reach nonvanishing values of $M$ (there is a transition from unbroken to broken phase). However, the values of $M$ for a fixed value of $\omega$ are smaller as the size of the

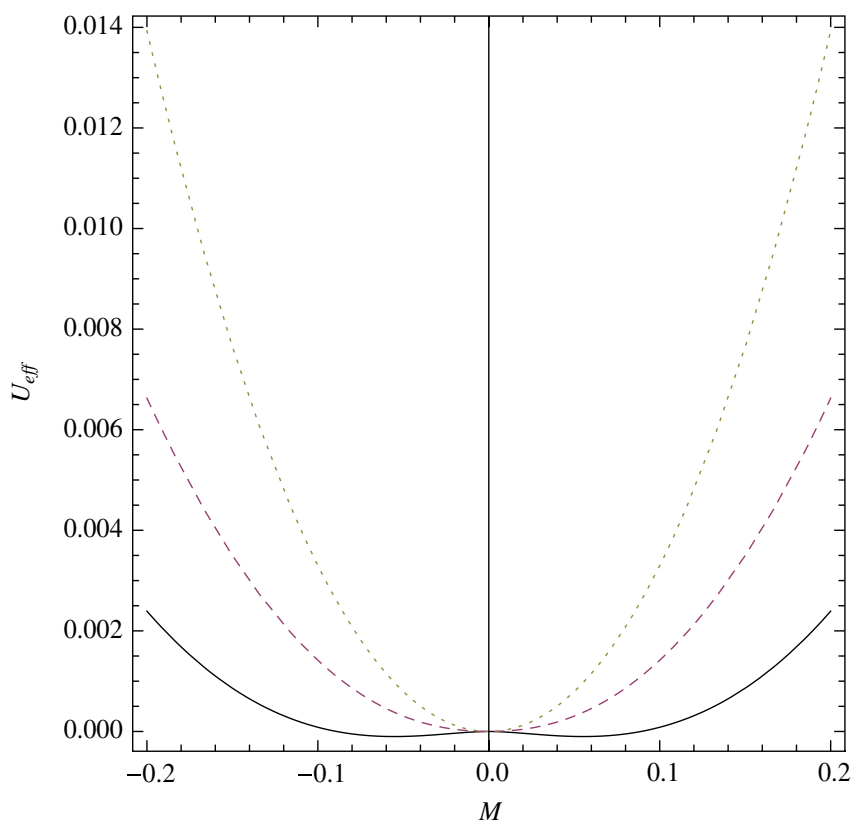

FIG. 8 (color online). Plot of effective potential, Eq. (20), for $T=0.01, \mu=0, G=\pi^{2} / 12$, and $\omega=1.0$. Solid, dashed, and dotted lines represent $x=0.01,0.5$, and 1.0 , respectively. 


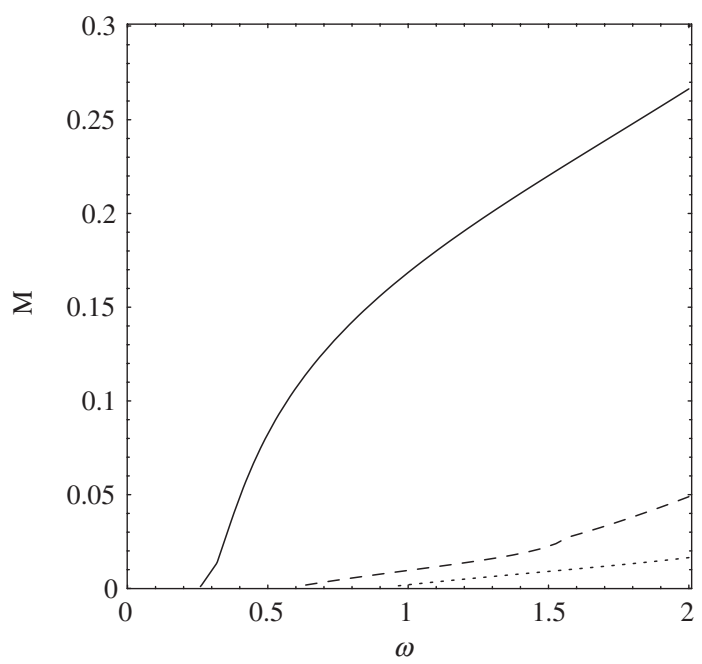

FIG. 9. Plot of effective quark mass $M$ versus magnetic field from Eq. (24) for $T=0.5, \mu=0.5$, and $G=\pi^{2} / 12$. Solid, dashed, and dotted lines represent $x=0.01,0.5$, and 1 , respectively.

system diminishes (the broken phase is inhibited, since smaller values of $M$ are reached).

In the plots of the effective potential shown above, we see that the nature of phase transition is of second order. For the model we treat, within the range of parameters we choose (even considering very high values of the magnetic field), no first-order transition is found for the system with a finite size.

It is also relevant to consider the dependence of the constituent quark mass on the size of the system. In Fig. 10 we plot $M$ versus $x=1 / L$ for two different values of magnetic field. We find that, for small values of $x$ (large system), $M$ increases with $\omega$, as expected from previous

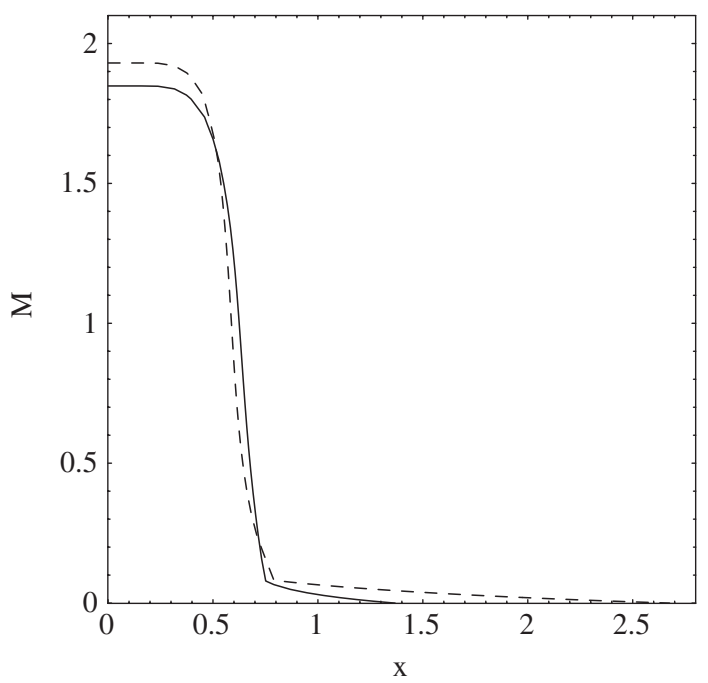

FIG. 10. Plot of effective quark mass $M$ versus inverse of size $x=1 / L$ from Eq. (24) for $T=0.01, \mu=0$, and $G=\pi^{2} / 2$. Solid and dashed lines represent $\omega=0.1$ and 1.0, respectively. results; this is another evidence of the magnetic catalysis. Increasing $x$, that is making more relevant the presence of boundaries, $M$ diminishes as the size $L$ decreases, with the order parameter vanishing for a finite value of $L$. Such a behavior clearly indicates the existence of a minimum size of the system below which the chiral symmetry is never broken.

Another relevant estimate is the dependence of critical temperature of the chiral phase transition on the size $L$ of the system; this is plotted in Fig. 11 for two different values of magnetic field. We find that the critical temperature decreases as the size of the system diminishes, a fact that also corroborates the statement that there exists a minimum value of $L$ for the chiral transition to be maintained; no finite solution of the gap equation, Eq. (24), can be found if one takes $L$ smaller than such a minimum value. Notice that, as indicated in Figs. 10 and 11, these minimum values are smaller for larger magnetic fields.

To estimate the size of the system below which the broken phase does not exist one needs to choose the value of the cutoff parameter $\Lambda$. For example, fixing $\mu=0$, $G=\pi^{2} / 2$, and $\omega=0.1$, we see from Figs. 10 and 11 that the maximum value of $x$ (measured in units of $\Lambda$ ) is $x_{\max } \approx 1.37$, corresponding to $L_{\min } \approx 0.74 \Lambda^{-1}$. Choosing $\Lambda=0.56 \mathrm{GeV}$, as suggested in Ref. [19], we find $L_{\text {min }} \approx 1.3 \mathrm{GeV}^{-1} \approx 0.3 \mathrm{fm}$. Thus, we can infer that the value of minimal size increases for $\omega<0.1$ (see Figs. 10 and 11), approaching 1 or a few fermi.

Nevertheless, it is important to emphasize the intricate phase structure of this system; it can be understood only by taking into account the set of parameters $(\mu, T, L, \omega)$, since for different values of this set one observes distinct behaviors for the phase diagram.

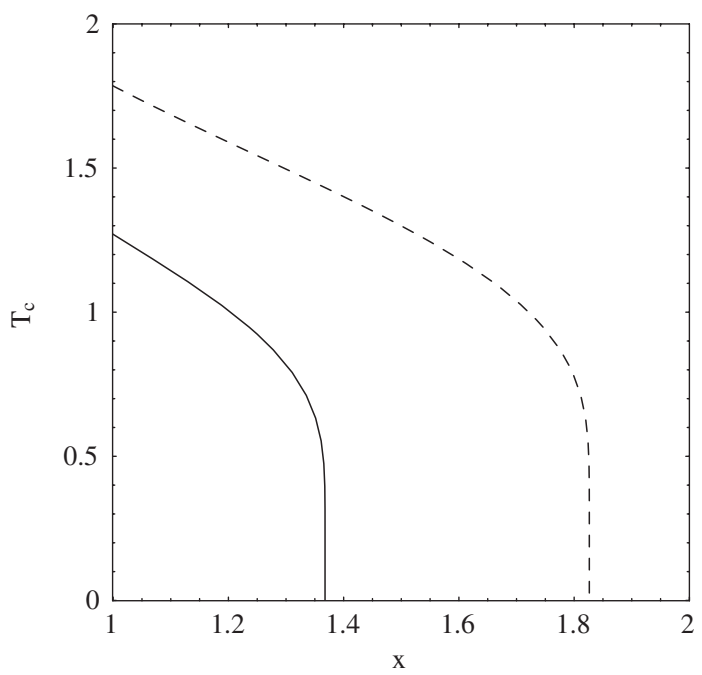

FIG. 11. Plot of critical temperature $T_{c}$ versus inverse of size $x=1 / L$ from Eq. (24) for $\mu=0$ and $G=\pi^{2} / 2$. Solid and dashed lines represent $\omega=0.1$ and 0.4 , respectively. 


\section{CONCLUSIONS}

In this work we have investigated the finite-size effects on the chiral transition in the framework of the NambuJona-Lasinio model, at finite temperature and density and in the presence of an external magnetic field. The thermodynamic potential and the gap equation have been obtained in the mean-field approximation. The behavior of the effective potential and solutions of the gap equation have been investigated under the change of the relevant parameters, with emphasis on the finite-size effects on the phase structure of the model.

Some points should be emphasized. One of them is that for given values of the chemical potential and magnetic field, the appearance of the broken phase is inhibited as the size of the system decreases, that is, decreasing the size of the system makes difficult the maintenance of long-range correlations and favors the disorder. Moreover, there exists a minimal size of the system below which the transition ceases to exist, in other words, the chiral-condensate phase cannot be sustained below this minimal size. These aspects are illustrated in Figs. 10 and 11, where are plotted, respectively, the effective quark mass and the critical temperature as functions of the inverse of the size of the system, at zero chemical potential, for two values of the applied magnetic field.

Another effect is that of enhancement of the broken phase as the magnetic field increases, which also occurs for the system with finite size, i.e., the effective mass $M$ increases with $\omega$ at a fixed size of the system. However, we stress that the dependence of $M$ with the intensity of the magnetic field is modified as the size of the system diminishes. The corresponding values of the effective mass are smaller for decreasing values of the size $L$ (larger values of $x$ ).

\section{ACKNOWLEDGMENTS}

This work received partial financial support from CNPq (Brazilian National Research Council). One of us (A.P.C.M.) thanks FAPERJ (Brazil) for partial financial support.
[1] G. Altarelli and G. Parisi, Nucl. Phys. B126, 298 (1977).

[2] C. L. Basham, L. S. Brown, S. D. Ellis, and S. T. Love, Phys. Rev. D 17, 2298 (1978).

[3] G. Preparata and J. Soffer, Phys. Rev. Lett. 61, 1167 (1988).

[4] V. Rivasseau, From Perturbative to Constructive Renormalization (Princeton University, Princeton, NJ, 1991), p. 54.

[5] J. C. Le Guillou and J. Zinn-Justin, Phys. Rev. B 21, 3976 (1980).

[6] E. J. Weniger, Phys. Rev. Lett. 77, 2859 (1996).

[7] U. D. Jentschura, Phys. Rev. A 64, 013403 (2001).

[8] S. A. Gottlieb, J. Kuti, D. Toussaint, A. D. Kennedy, S. Meyer, B. J. Pendleton, and R. L. Sugar, Phys. Rev. Lett. 55, 1958 (1985).

[9] M. D'Elia, S. Mukherjee, and F. Sanfilippo, Phys. Rev. D 82, 051501 (2010).

[10] P. V. Buividovich, M. N. Chernodub, E. V. Luschevskaya, and M. I. Polikarpov, Phys. Rev. D 81, 036007 (2010).

[11] P. V. Buividovich, M. N. Chernodub, E. V. Luschevskaya, and M. I. Polikarpov, Phys. Lett. B 682, 484 (2010).

[12] P. V. Buividovich, M. N. Chernodub, E. V. Luschevskaya, and M. I. Polikarpov, Nucl. Phys. B826, 313 (2010).

[13] O. Bergman, G. Lifshitz, and M. Lippert, J. High Energy Phys. 05 (2008) 007.

[14] C. V. Johnson and A. Kundu, J. High Energy Phys. 12 (2008) 053.

[15] A. V. Zayakin, J. High Energy Phys. 07 (2008) 116.

[16] Y. Nambu and G. Jona-Lasinio, Phys. Rev. 122, 345 (1961); 124, 246 (1961).

[17] S. P. Klevansky, Rev. Mod. Phys. 64, 649 (1992).

[18] T. Hatsuda and T. Kunihiro, Phys. Rep. 247, 221 (1994).
[19] M. Buballa, Phys. Rep. 407, 205 (2005).

[20] D. Ebert and K. G. Klimenko, Nucl. Phys. A728, 203 (2003).

[21] T. Inagaki, D. Kimura, and T. Murata, Prog. Theor. Phys. 111, 371 (2004).

[22] K. Fukushima, D. E. Kharzeev, and H. J. Warringa, Phys. Rev. D 78, 074033 (2008).

[23] E. S. Fraga and A.J. Mizher, Phys. Rev. D 78, 025016 (2008).

[24] D. P. Menezes, M. B. Pinto, S. S. Avancini, A. P. Martínez, and C. Providência, Phys. Rev. C 79, 035807 (2009).

[25] Sh. Fayazbakhsh and N. Sadooghi, Phys. Rev. D 83, 025026 (2011).

[26] S. Chakrabarty, J. Astrophys. Astron. 16, 399 (1995).

[27] A. J. Mizher, M. N. Chernodub, and E. S. Fraga, Phys. Rev. D 82, 105016 (2010).

[28] R. Gatto and M. Ruggieri, Phys. Rev. D 82, 054027 (2010).

[29] R. Gatto and M. Ruggieri, Phys. Rev. D 83, 034016 (2011).

[30] S. P. Klevansky and R. H. Lemmer, Phys. Rev. D 39, 3478 (1989).

[31] H. Suganuma and T. Tatsumi, Ann. Phys. (N.Y.) 208, 470 (1991).

[32] I. A. Shushpanova and A. V. Smilga, Phys. Lett. B 402, 351 (1997).

[33] D. N. Kabat, K. M. Lee, and E. J. Weinberg, Phys. Rev. D 66, 014004 (2002).

[34] T. Inagaki, D. Kimura, and T. Murata, Prog. Theor. Phys. 111, 371 (2004).

[35] T. D. Cohen, D. A. McGady, and E. S. Werbos, Phys. Rev. C 76, 055201 (2007). 
[36] V.P. Gusynin, V. A. Miransky, and I. A. Shovkovy, Nucl. Phys. B462, 249 (1996).

[37] V.P. Gusynin, V. A. Miransky, and I. A. Shovkovy, Nucl. Phys. B563, 361 (1999).

[38] G. W. Semenoff, I. A. Shovkovy, and L.C.R. Wijewardhana, Phys. Rev. D 60, 105024 (1999).

[39] V. A. Miransky and I. A. Shovkovy, Phys. Rev. D 66, 045006 (2002).

[40] K. G. Klimenko, Theor. Math. Phys. 89, 1161 (1991).

[41] K. G. Klimenko, Theor. Math. Phys. 89, 1287 (1991).

[42] K. G. Klimenko, Z. Phys. C 54, 323 (1992).

[43] K. G. Klimenko, Theor. Math. Phys. 90, 1 (1992).

[44] B. Hiller, A. A. Osipov, A. H. Blin, and J. da Providencia, Sigma 4, 024 (2008).

[45] N. O. Agassian and S. Fedorov, Phys. Lett. B 663, 445 (2008).

[46] D. K. Kim, Y.D. Han, and I. G. Koh, Phys. Rev. D 49, 6943 (1994).

[47] Y. B. He, W. Q. Chao, C. S. Gao, and X. Q. Li, Phys. Rev. C 54, 857 (1996).

[48] A. S. Vshivtsev, M. A. Vdovichenko, and K. G. Klimenko, J. Exp. Theor. Phys. 87, 229 (1998).

[49] J. B. Kogut and C. G. Strouthos, Phys. Rev. D 63, 054502 (2001).

[50] O. Kiriyama and A. Hosaka, Phys. Rev. D 67, 085010 (2003).
[51] C. G. Beneventano and E. M. Santangelo, J. Phys. A 37, 9261 (2004).

[52] A. V. Gamayun and E. V. Gorbar, Phys. Lett. B 610, 74 (2005).

[53] O. Kiriyama, T. Kodama, and T. Koide, arXiv:hep-ph/ 0602086.

[54] D. Ebert, K. G. Klimenko, A. V. Tyukov, and V.C. Zhukovsky, Phys. Rev. D 78, 045008 (2008).

[55] L. M. Abreu, M. Gomes, and A. J. da Silva, Phys. Lett. B 642, 551 (2006).

[56] L. M. Abreu, A.P. C. Malbouisson, J. M. C. Malbouisson, and A. E. Santana, Nucl. Phys. B819, 127 (2009).

[57] L. M. Abreu, A.P.C. Malbouisson, and J.M.C. Malbouisson, Europhys. Lett. 90, 11001 (2010).

[58] L. M. Abreu, A.P.C. Malbouisson, and J.M.C. Malbouisson, Phys. Rev. D 83, 025001 (2011).

[59] E. Elizalde, Ten Physical Applications of Spectral Zeta Function, Lecture Notes in Physics (Springer-Verlag, Berlin, 1995).

[60] M. Frasca and M. Ruggieri, Phys. Rev. D 83, 094024 (2011).

[61] V. I. Ritus, Ann. Phys. (N.Y.) 69, 555 (1972).

[62] C. N. Leung and S. Y. Wang, Nucl. Phys. B747, 266 (2006).

[63] L. M. Abreu, C. de Calan, A. P. C. Malbouisson, J. M. C. Malbouisson, and A. E. Santana, J. Math. Phys. (N.Y.) 46, 012304 (2005). 